\title{
Le conte de fées de Gotlib. Entre hommage, parodie et transgression
}

Alain Corbellari

\section{(2) OpenEdition \\ 1 Journals}

Édition électronique

URL : https://journals.openedition.org/edl/1644

DOI : $10.4000 /$ edl. 1644

ISSN : 2296-5084

Éditeur

Université de Lausanne

\section{Édition imprimée}

Date de publication : 15 septembre 2019

Pagination : 189-206

ISBN : 978-2-940331-71-0

ISSN : 0014-2026

\section{Référence électronique}

Alain Corbellari, «Le conte de fées de Gotlib. Entre hommage, parodie et transgression », Études de lettres [En ligne], 310 | 2019, mis en ligne le 15 septembre 2021, consulté le 22 septembre 2021. URL: http://journals.openedition.org/edl/1644; DOI : https://doi.org/10.4000/edl.1644

Ce document a été généré automatiquement le 22 septembre 2021.

(c) Études de lettres 


\title{
Le conte de fées de Gotlib. Entre hommage, parodie et transgression
}

\author{
Alain Corbellari
}

1 On a vite fait de réduire l'œuvre de Marcel Gotlib (1935-2016) à sa dimension parodique et, certes, l'apport majeur de ce géant de la bande dessinée au renouveau de son art réside bien dans son adaptation au cadre francophone des procédés de dérision développés aux USA par le magazine Mad et les cartoonists américains d'avant-garde des années 1960. Cependant, une telle caractérisation ne rend que fort imparfaitement compte de l'extraordinaire variété des formes que prend, au-delà de la simple parodie, la pulsion intertextuelle et multiréférentielle qui se fait jour dans le travail de Gotlib. On constate par ailleurs très vite que délimiter des axes thématiques au sein de cette nébuleuse graphique qui a profondément marqué la bande dessinée francophone s'avère à la fois évident, tant la récurrence de certains thèmes et procédés est visible, et fallacieux, dans la mesure, précisément, où les hybridations thématiques et génériques font partie intégrante des techniques de distorsion gotlibiennes.

2 Cerner les usages que Gotlib fait du conte de fées passe ainsi par l'examen de son rapport à l'enfance, rapport qui s'avère essentiel dans la production de notre auteur et qui dépasse de beaucoup, comme on s'en doute, la simple question de la parodie. De fait, la problématique de l'enfance se décline au moins sur trois plans qui représentent autant de champs de tension conflictuels, constituant les traits définitoires de ses bandes dessinées.

3 1) S'impose tout d'abord le rapport «autobiographique » (ou «autofictionnel») de l'auteur à sa propre enfance. Enfant juif ayant passé la guerre chez des paysans français tandis que son père allait mourir à Buchenwald, Gotlib développe dans plusieurs épisodes de la Rubrique-à-brac (désormais $R A B$ ) une description nostalgique et (parfois fallacieusement) idyllique de ses souvenirs d'enfance ${ }^{1}$. C'est le cas en particulier de "Sur l'air du tra-deri-dera » ou "Chanson aigre-douce " ${ }^{2}(R A B, 2,58-59)$ où il présente son enfance apparemment paisible à la campagne, rythmée par une comptine où il est question d'un "orage", dont il ne comprendra que plus tard qu'il symbolisait la Guerre. Cette référence explicite, et faite sur un ton sérieux, à un contexte 
biographique précis reste cependant exceptionnelle ; on assiste d'habitude plutôt à des mises en scène dont l'ancrage référentiel réel est sinon gommé, du moins évoqué de biais. Dans «L'époque bénie de l'initiation» (RAB, 1, 94-95), le narrateur évoque avec tendresse le «boueux de [s]on enfance » en transfigurant la trivialité du ramassage des ordures sur le ton de la geste héroïque. Dans «Chanson rose, chanson mauve » $(R A B, 1$, 16-17), il met en scène un enfant qui enchante les moindres gestes de la vie quotidienne et qui fait soudain des "découvertes étranges ", l'amenant progressivement à l'idée que les choses ne sont peut-être rien de plus que ce qu'elles sont (il n'y a plus d'oiseau dans l'appareil photo, ni de précipice au bord du trottoir, ni de lutin au bout des doigts), prélude à l'accession à la maturité, évoquée par une pirouette finale douce-amère : « les adultes sont de grands gosses, mais ce ne sont plus des enfants ». Enfin, cas-limite, qui ne peut être autobiographique au sens strict, mais ressortit bien à une forme d'autofiction, "Conquête » ou " Journal d'un conquistador » (RAB, 2, 32-33), raconte, sur le mode du récit d'un explorateur et en caméra subjective, les premières semaines d'un bébé. Ici encore, une authentique tendresse poétique l'emporte sur la dérision.

Cette focalisation sur l'enfance se retrouvait par ailleurs déjà souvent dans les Dingodossiers, qui avaient précédé la $R A B$ et dont Gotlib n'était que le dessinateur, puisque leurs scénarios étaient dus à René Goscinny ${ }^{3}$. Ce dernier, en proposant à Gotlib d'illustrer cette série, avait probablement deviné chez son jeune collaborateur une affinité spéciale pour le monde de l'enfance, car on imagine mal que Goscinny ait pu déterminer à lui seul cette composante essentielle de l'œuvre gotlibienne. D'ailleurs, de manière significative, l'enfance dans les Dingodossiers est vue à travers la quotidienneté de l'existence des enfants et pour ainsi dire jamais à travers la production culturelle qui leur est dédiée; ainsi les contes de fées en sont-ils totalement absents, signe que la propension à les mettre en scène relève bien d'un élément d'imaginaire purement gotlibien. Plus porté à la description drolatique de la vie moderne ${ }^{4}$, Goscinny ne l'était en effet qu'assez peu à la réécriture des classiques de notre culture. Sa rencontre n'en aura pas moins été essentielle pour Gotlib, qui n'aura de cesse de mettre cette référence à distance (faisant en particulier de Goscinny - dans un geste éminemment freudien ${ }^{5}$ le coupable systématique des aventures policières de Charolles et Bougret), mais qui ne reniera, en fait, jamais sa dette envers l'auteur du Petit Nicolas. Certains des premiers épisodes de la $R A B$ se situent ainsi dans la ligne directe des Dingodossiers, tels «Prospective» (RAB, 1, 88-89), fantaisie sur l'évolution télécommandée des plus archaïques jeux enfantins ou " Mise en garde » $(R A B, 1,46-47)$ qui liste les déformations physiques indésirables causées par les activités de vacances (avec, cependant, une automise en scène finale typiquement gotlibienne, où l'on voit le dessinateur défiguré par les imprudences qu'il a commises lors de la pause estivale).

2) Vient ensuite le rapport "professionnel » de l'auteur au medium dans lequel il s'exprime, medium qui connaît précisément au moment où la carrière de Gotlib prend son envol (fin des années 1960) une évolution vers une saisie " adulte » de ses tenants et aboutissants à travers un processus dont la revue Pilote, où il publie ses travaux les plus emblématiques (et nous retrouvons ici encore la figure incontournable de René Goscinny), sera précisément le fer de lance ${ }^{6}$.

6 Notons que la réflexion gotlibienne sur l'accession de la $\mathrm{BD}$ à une forme de maturité interfère avec sa propension à la mise en scène de soi. Ainsi Gotlib aime-t-il se présenter, surtout à partir du quatrième tome de la $R A B$, sous les traits d'un « artiste » couronné de lauriers (voir "Croyez-en ma vieille expérience", RAB, 4, 10-11, ou 
«Physiologie », $R A B, 4,38-39)$, mais « Comment il naquit » (RAB, 2, 4-5), sur la naissance du personnage gotlibien d'Isaac Newton, parodiait déjà la pose de l'artiste maudit.

3) Il y a enfin le rapport «critique » de l'auteur à la tradition même de la littérature dite " enfantine ». C'est évidemment ce dernier rapport qui sera au cœur de la présente contribution; mais il convient de ne pas perdre de vue les deux premiers, qui entrent nécessairement en dialogue avec le troisième, la dimension autofictionnelle étant omniprésente dans l'œuvre de Gotlib et la question du support restant cruciale dans l'optique du dessinateur.

8 Les contes de fées sont présents de très nombreuses manières chez Gotlib : ils peuvent être le support explicite d'un épisode entier, être évoqués à titre de citations ponctuelles ou bien à travers leurs topoï récurrents. Encore ces trois possibilités sontelles loin de rendre compte de la variété des usages pratiqués par Gotlib qui, contrairement à nombre de parodistes modernes, réinvente à chaque fois son angle d'attaque: rien de plus éloigné de Gotlib que la manière d'un Garner qui, une fois trouvé le truc de la réécriture "politiquement correcte", use le procédé jusqu'à la corde ${ }^{7}$. Il convient aussi d'évoquer séparément les usages faits du conte de fées dans la $R A B$ et ceux que le dessinateur se permettra plus tard: aussi irrespectueuses que puissent paraitre certaines parodies de la $R A B$, elles restent toujours regardables par des enfants, ce qui ne sera plus le cas des allusions disséminées dans les albums ultérieurs. On commencera donc (réservant les usages tardifs pour une comparaison conclusive) par lister les épisodes de la RAB que l'on peut directement lier aux contes de Perrault, de Grimm et d'Andersen.

Cette sélection d'épisodes demeure cependant quelque peu arbitraire: que faire des fables de La Fontaine ${ }^{8}$, de récits comme Pinocchio ou Alice au pays des merveilles ${ }^{9}$ ou des récits où interviennent des fées ou des sorcières sans référence directe à des contes répertoriés? Sans compter que Gotlib traite volontiers à la manière des contes certains récits célèbres de la littérature populaire, en particulier celui de Tarzan, souvent sollicité dans la $R A B$, parfois en compagnie de Mowgli, lequel entretient lui-même des liens évidents avec le thème de L'Enfant sauvage de Truffaut, qui est l'objet d'un épisode du tome 3 (20-23); ce qui nous entraîne du côté des parodies cinématographiques, omniprésentes dans la $R A B$. De fait, les épisodes jouant sur des variations autour de topoï narratifs sont aussi caractéristiques des reprises de film que des reprises de contes chez Gotlib. Par ailleurs, plusieurs épisodes de la RAB trouvent leur source dans les traditions populaires, les comptines et le monde paysan, tous éléments qui entretiennent ici encore des rapports certains avec l'univers du conte de fées. Et que dire, pour passer du conte à l'histoire drôle, des multiples variations sur la «blague du fou qui repeint son plafond »?

10 On ne pouvait évidemment résumer ici toutes ces déclinaisons du détournement de la pulsion narrative, et l'on retiendra donc dans notre corpus strict :

- « Histoire à considérer sous plusieurs angles » (RAB, 1, 8-9), deuxième épisode de la publication en album de la $R A B$, peut éventuellement rappeler certains éléments des «Fées » de Perrault; c'est une variation sur le thème du prince amoureux de la bergère : celle-ci, sublime de face, s'avère laide de profil, et c'est seulement dans la dernière image, au moment où le prince, déçu et énervé, se tourne vers le lecteur pour lui faire remarquer qu' " on devrait toujours voir les gens de face et de profil à la fois ", que l'on s'aperçoit que le protagoniste, toujours vu jusque là sous son beau profil, possède une face difforme! 
- « Sade raconté aux enfants » $(R A B, 1,14-15)$ : reprise littérale (!) de larges passages du "Petit Poucet", assortis de commentaires essentiellement destinés à souligner la cruauté du texte de Perrault. C'est la seule fois que l'on surprend Gotlib livre en main : à l'évidence, l'épisode superpose à des souvenirs du conte remontant à l'enfance une relecture du texte original faite par l'adulte, lequel s'avoue consterné de découvrir que, loin d'être une simple et gentille histoire à mettre entre toutes les mains, le conte de Perrault, terriblement retors dans ses formulations, est l'œuvre d'un écrivain très sûr de ses moyens. On ne retrouvera nulle part ce retour au texte, toutes les autres réminiscences gotlibiennes de contes pouvant s'expliquer par le simple recours à la mémoire des lectures enfantines.

- «Les beaux contes de notre enfance » et « Les beaux contes de notre enfance (suite et fin) », respectivement «Le vilain petit canard » et «Le retour du vilain grand cygne » $(R A B, 1,18-21)$ : reprise humoristique mais relativement fidèle du conte d'Andersen (sinon que Gotlib précise qu'en grandissant le petit cygne n'est pas devenu beau pour autant !), suivie d'une continuation faisant bifurquer le conte sur celui de « La Belle et la Bête », le vilain grand cygne voulant devenir un prince étant transformé...en héros de ce dernier conte.

- «Une ordonnance maladroite » ou «La triste histoire du loup végétarien marqué par son hérédité » $(R A B, 1,38-39)$ : reprise rapide de cinq contes et fables mettant en scène un loup («Le loup et l'agneau », "Pierre et le loup », "La chèvre de Monsieur Seguin », "Les trois petits cochons » et "Le Petit Chaperon rouge ») en installant à chaque fois le loup dans une position de vaincu, puisqu'il s'agit des cauchemars d'un loup végétarien ; le médecin conseillant au loup de changer de régime sera évidemment la première victime de l'animal rendu à ses instincts naturels.

- "Gadgets pour contes de fées" $(R A B, 1,50-51)$ : neuf objets allant de la paire de jumelles pour sœur Anne à la bombe insecticide pour Pinocchio (pour se débarrasser de sa conscience), en passant par la machine à coudre pour la Belle au bois dormant ou le bicarbonate pour Blanche-Neige, et grâce auxquels le déroulement des contes aurait pu être infléchi.

- "Supposition idiote" $(R A B, 1,32-53)$ : à savoir «si les pommes étaient des citrouilles ». Parmi les exemples, celui, évidemment, de Blanche-Neige.

- «Une erreur grossière » ou "l'examen de magie » (RAB, 1, 64-65) : apprentie-fée, Caroline est recalée pour avoir rendu bête un prince laid mais intelligent (souvenir de "Riquet à la houppe ») et laide une bergère belle mais stupide au lieu, évidemment, d'avoir rendu beau le premier et intelligente la seconde.

- « Reconversion » $(R A B, 1,74-75)$ : ou comment l'ogre, humilié par le « Petit Poucet » et quitté par sa femme, devient le Père Noël.

- "Réflexions non dénuées d'intérêt» $(R A B, 1,84-85)$ : au milieu de réflexions extrêmement hétéroclites, on voit qu'il suffit au Petit Poucet de se jucher sur une pile de dictionnaires pour avoir l'air, derrière un talus d'où n'émergent que les têtes, plus grand que ses autres frères $!^{10}$

- «Le Scénario refusé », fable-express $(R A B, 2,25)$ : un scénariste propose l'histoire de «Blanche-Neige » à un producteur qui lui fait remarquer que cette histoire a déjà été écrite. Moralité : « le Grimm ne paie pas ».

- "Au temps des fées" ou "L'humble paysan victime d'une plaisanterie de mauvais goût (à son grand dam) » $(R A B, 2,34-35)$ : un paysan se montre ostensiblement bon avec une grenouille (souvenir du "Roi-Grenouille») en espérant bien que sa bonté sera remarquée par une bonne fée. Mais la grenouille est attachée à une ficelle tirée par un 
gamin. Vexé, le paysan corrige le gamin...qui se révèle malheureusement être une fée déguisée, laquelle, pour punir le paysan, le transforme en grenouille.

- «Continuons sur la lancée » $(R A B, 2,60-61)$ : huit variations de plus en plus délirantes sur le thème des objets semés par le «Petit Poucet " pour retrouver le chemin de la maison.

- «Le passeur malhonnête» (RAB, 2, 73), fable-express : sœur Anne, traversant la rivière pour voir de plus près si les deux frères arrivent, se fait escroquer par un batelier. Moralité : « Sœur Anne aux deux berges raque ».

- «Trop parler nuit » ou « le vieux pêcheur oublieux» $(R A B, 3,16-17)$ : pour avoir aidé un " kerlutinhoëc ", un vieux pêcheur reçoit chaque jour un énorme tas de poissons (on peut éventuellement songer aux contes de pêcheurs des Grimm). Il oublie malheureusement qu'il ne fallait raconter cette histoire à personne, sous peine de voir le charme se rompre.

- "Y'en a être une fois... » (RAB, 3, 55-56) : récriture du «Petit Chaperon rouge » en mode « conte africain » avec détails empruntés au " Petit Poucet » et à " Peau d'âne ».

- «Trou de mémoire » $(\mathrm{RAB}, 3,68-69)$ : le narrateur tente à six reprises de raconter « Le Petit Chaperon rouge ». Comme il n'y arrive décidément pas, c'est l'héroïne, relayée par le loup, bonne pâte et pipe au bec, qui finit par résumer le conte. Dans la dernière case, la grand'mère vient chercher elle-même le panier, parce qu'elle "commence à la sauter ", tandis que le loup, en arrière-plan, demande au Petit Chaperon rouge si elle n'aurait pas par hasard un moment de libre : Gotlib se souviendra de cette conclusion ouverte dans une histoire tardive que l'on examinera un peu plus loin.

- «Sac de nœuds chez les princes » $(R A B, 5,17-19)$ : les princes charmants de «BlancheNeige ", "Cendrillon » et "Peau d'âne » se retrouvent au chevet de «La Belle au bois dormant » et se font éconduire sans ménagement par le prince officiel.

- «Le genre d'accident bête » ou "Incident de parcours » $(R A B, 5,22-23)$ : un paysan médiéval (le même que dans "Au temps des fées »?) libère une fée minuscule d'une toile d'araignée : désireuse de le récompenser, elle se fait malheureusement gober par un brochet.

11 On constate qu'une part non négligeable de ces épisodes (et la proportion s'accroît encore si on y adjoint les récits " para-féeriques ») est concentrée dans le premier tome de la $R A B$, paru en 1970 (pour des histoires parues dans Pilote en 1968) et que l'on n'en voit presque plus dans les trois derniers. Il y a donc bel et bien un éloignement progressif de Gotlib par rapport à un certain "esprit d'enfance » de sa production, même si les rares exemples qu'offre le cinquième tome ne sont pas, contrairement à d'autres épisodes du même volume où l'on voit une nette évolution vers un langage et des thématiques plus adultes, d'un ton très différent des contes réunis dans le premier tome. Cependant, quand Gotlib reviendra aux contes dans Rhââ-Lovely, le dynamitage sera consommé!

Certaines récurrences thématiques s'observent. La question de la laideur et de la beauté (liée tant à l'histoire de "Riquet à la houppe " qu'à celle du "Vilain petit canard ») permet par exemple de mettre en cause les codes de la narration graphique : dans "Histoire à considérer sous plusieurs angles", c'est sur sa propre propension à représenter majoritairement ses personnages complètement de profil ou complètement de face - alors que les autres dessinateurs, à la technique plus souple, privilégient massivement le trois-quarts-face - que joue Gotlib; les jeunes fées plus ou moins maladroites (redoublement dans le cadre de la littérature enfantine du thème de 
l'immaturité) et, au niveau des sujets, «Le Petit Poucet » et « Le Petit Chaperon rouge » se taillent (si l'on ose dire !) la part du lion.

Quant à la question de la narration, elle est omniprésente : à certains égards, toute la $R A B$ apparait comme une vaste réflexion sur le problème du récit et de ses limites. Particulièrement emblématique s'avère, à cet égard, l'épisode intitulé "Aller-retour " $(R A B, 4,58-59)$, qui ne nous éloigne guère de notre sujet puisqu'il met en scène la (soidisant) récitation d'un conte : on y voit "Oncle Gotlib " (allusion aux « belles histoires de l'Oncle Paul » dont les récits didactiques se sont égrenés des années 1950 aux années 1970 dans le journal de Spirou) s'apprêtant à raconter à un groupe d'enfants l'histoire que lui racontait sa grand-mère, laquelle la tenait d'un vagabond, qui la tenait de son maitre d'apprentissage, qui la tenait d'un officier anglais de l'armée des Indes, qui la tenait d'un vieux prêtre hindou, qui la tenait d'un Indien d'Amérique,...lequel buvait et n'avait par conséquent jamais réussi à se souvenir de l'histoire en question. Le temps que cette information déceptive remonte la chaîne en sens inverse, l'épisode de la semaine est bouclé, bien que Gotlib ne se console pas d'avoir oublié cette histoire "si belle pourtant que [lui] contait [sa] grand-mère", à l'évocation de qui il fond en sanglots. Il n'y a donc pas d'histoire, mais le désir d'histoire est si fort qu'il provoque exactement les mêmes affects que si l'histoire avait existé. Du moins - faut-il s'empresser de le préciser - chez le narrateur, car les enfants, blasés et s'échangeant des cigarettes (!), s'en vont en jugeant que l'histoire du jour n'est "pas terrible ", " manque de chute » et que «le développement est faible». Sur quoi, "Oncle Gotlib», vexé, leur reproche vertement de manquer de "respect pour les aînés ». La " moralité » de l'épisode est donc ambiguë : le narrateur se plaint-il, sur un mode nostalgique, qu'il n'y ait «plus de jeunesse » ou raille-t-il la propension des aînés à ressasser un passé sans intérêt? Comme aucune histoire n'a été racontée, tout jugement sur le contenu de celle-ci reste impossible.

Sans intellectualiser à outrance les planches de Gotlib, il n'est pas indifférent de rappeler que la $R A B$ est contemporaine de l'apogée du structuralisme ${ }^{11}$ et que le soupçon que notre auteur fait peser - de toutes les manières possibles - sur la possibilité même du récit est évidemment, par ailleurs, l'un des plus sûrs moyens qu'il ait trouvés pour faire accéder le médium BD à l'âge de raison. J'y reviendrai en conclusion.

Reprenons pour l'heure les épisodes constituant notre corpus proprement dit. Peut-on déterminer un angle d'attaque privilégié de Gotlib ? Pas vraiment, et une catégorisation trop rigide risquerait de nous faire oublier que plusieurs procédés peuvent être utilisés concurremment dans la même histoire. Le dessinateur peut jouer sur la reprise bifurquée (les conclusions inattendues du «Vilain petit canard» ou du "Savetier et le financier ») ou sur les variations sérielles (les objets semés par le « Petit Poucet»). C'est tantôt un conte précis, tantôt les topoï d'un imaginaire générique plus flou qui sont sollicités: jusqu'où peut-on considérer que l'apparition d'une fée est une allusion directe aux "contes de fées»? Les hybridations peuvent être intra- ou intergénériques $^{12}$ : "Sac de nœuds chez les princes" se maintient dans la mouvance de Perrault, tandis que «Gadgets pour contes de fées » explore un spectre plus vaste. Un même épisode peut réinterpréter différemment l'axiologie des récits qu'il utilise : ainsi l'histoire sérielle du loup végétarien juxtapose-t-elle inversions (c'est le loup qui trouble l'« onde pure » de l'agneau ou qui est vaincu par le chèvre de Monsieur Seguin) et confirmations (la bastonnade infligée au loup par le Petit Chaperon rouge n'entre pas 
en contradiction totale avec la conclusion traditionnelle du conte). Enfin, le conte de fées peut lui-même être totalement périphérique dans une rubrique fourre-tout qui ne le sollicite pas de prime abord, comme dans « Réflexions non dénuées d'intérêt ».

À titre d'exemple, on s'attardera un peu plus longuement ici sur "Trou de mémoire ", qui pousse à la perfection le procédé gotlibien de la variation sur un motif narratif.

La première bande est destinée à présenter l'héroïne, à savoir le Petit Chaperon rouge, dont le narrateur (toujours en voix off) commente l'habillement; il n'est pas indifférent, à cet égard, que le capuchon à franges crénelées dont il l'affuble soit le même dont il vêt ses paysans du Moyen Âge. La suggestion, dans la troisième case, de donner à l'héroïne un habillement de style Renaissance n'est finalement pas retenue, ce qui montre bien le lien privilégié que Gotlib tisse entre imaginaire médiéval et conte de fée.

Le récit commence dans la deuxième bande, pour s'interrompre immédiatement, le narrateur se rendant vite compte que l'idée de faire intervenir l'ogre est inadéquate. Dans la troisième bande, c'est carrément le blanc, et ce n'est finalement qu'au troisième recommencement, dans la quatrième bande, que le narrateur se souvient que son héroïne rencontre... le loup ! Il en est si content qu'il décide de reprendre une fois de plus tout à zéro. Nous arrivons ici à la fin de la première des deux pages de l'histoire, et cette nouvelle décision de recommencer a d'autant plus valeur de césure qu'elle permet à la fois d'introduire le deuxième protagoniste du conte et de remonter le moral de l'héroïne, dont l'agacement de devoir toujours reprendre la scène commençait à devenir visible. Suivent, à la deuxième page, trois bandes $(5,6$ et 7) qui montrent successivement le loup volant le panier de l'héroïne, se faisant violemment frapper par celle-ci et, finalement, lui contant fleurette, avant que la fillette ne craque en hurlant un «MAIS NON » désespéré qui rompt le cycle des répétitions. La huitième et dernière bande du récit répond ainsi à la première pour encadrer les six bandes répétitives, dont elle clôt la compulsion obsessionnelle (en racontant la fin) tout en ouvrant le récit sur un possible (le loup et le Chaperon rouge entament un nouveau type de relation) tout aussi infidèle à Perrault que les six débuts avortés qui ont précédé.

19 Chacune des bandes répétitives se décline en quatre images : la première nous fait voir la mère donnant le panier à sa fillette, la deuxième, plus étroite, nous montre celle-ci entamant sa promenade, la troisième illustre l'erreur ou le blanc du narrateur, et la quatrième tente de remettre les pendules à l'heure. Gotlib varie la première image en plantant deux puis quatre aiguilles dans le chignon de la mère, puis en y introduisant un os, puis en la coiffant de pamirs, puis enfin en ne la faisant plus parler qu'en horschamp. Ces variations, qui sont autant de dégradations de l'image initiale, font sentir le temps qui passe, contredisant paradoxalement le sur-place du récit. De la même manière, dans la deuxième image, le Petit Chaperon rouge, successivement, chante gaiement, puis en forçant un peu sa joie, puis visiblement agacée, puis à nouveau de tout son cœur mais un peu trop théâtralement (haut de la deuxième page), puis d'un air excédé, et finalement en pleurant à chaudes larmes. La dégradation ici n'est plus seulement graphique, elle comporte une forte charge affective qui, une fois encore, superpose la temporalité de l'actrice forcée à refaire toujours les mêmes prises (la référence cinématographique est ici évidente) et le piétinement de l'action. Le loup, par contre, présent dans toutes les troisièmes et quatrièmes cases à partir de la troisième variation, ne présente que les affects exigés par le narrateur: enchanté quand ce dernier a enfin décidé de le faire intervenir, féroce et empressé quand il vole le panier, 
résigné lorsqu'il se fait cogner, enjôleur quand il doit séduire. Face à la crise de nerfs de l'héroïne (dont l'agacement est encore visible dans l'ultime case de l'épisode), il reste placide, parodiant, avec sa pipe, l'image du grand père débonnaire racontant des contes à la veillée ${ }^{13}$. Face à cette prise en charge imprévue de l'histoire par ses personnages, le narrateur, vexé, termine sur un boudeur «puisque c'est comme ça, je ne dirai plus rien ", clausule ironique qui ne fait, en fin de compte, que corroborer son incapacité à raconter quoi que ce soit. Feignant d'être dépassé par ses personnages, Gotlib ridiculise ici les vantardises hypocrites des romanciers qui prétendent que leurs personnages sont tellement bien imaginés qu'ils se sont mis à vivre "de leur propre vie ». Enfin, n'oublions pas la mère-grand, qui « commence à la sauter » et vient prendre son panier elle-même dans la dernière image.

Mais il faudrait aussi mentionner les interventions de la petite coccinelle qui vient continuellement parasiter les pages de la $R A B$ : on la voit ici voler furtivement la galette, puis le petit pot de beurre, avant de commenter le déroulement chaotique de l'histoire en la trouvant "dans le fond » tour à tour «brutale ", " plein[e] d'imprévu ", "pleine de bruit et de fureur» (référence à Shakespeare via Faulkner), "à rebondissements ", « triste » et " polissonne », avant de renoncer à la comprendre et de s’éclipser.

Cette mécanique complexe superpose ainsi plusieurs niveaux d'énonciation et même plusieurs régimes médiatiques : tout d'abord la voix du narrateur, que l'on pourrait dire purement littéraire, puisque Gotlib se garde bien de céder à son penchant à l'autoreprésentation graphique et qu'on pourrait même dire qu'il se situe clairement, avec ses pirouettes qui le décrédibilisent (ainsi de la conclusion), dans la lignée de qu'on a appelé le "récit excentrique ${ }^{14}$. Mais il s'agit en même temps d'une "voix off » qui nous entraîne du côté de la lecture cinématographique, rendue évidente par l'assimilation implicite des variations à la technique des reprises dues aux aléas d'un tournage filmique: les personnages deviennent ainsi des acteurs victimes de l'amateurisme d'un cinéaste particulièrement brouillon. Enfin, naturellement, la dialectique graphico-narrative du même et de l'autre ne prend toute sa signification que dans l'espace bédéique de la double planche, doublement saturé par les éléments récurrents (costumes, personnages, panier, etc.) et par les facéties parasitaires de la coccinelle.

Que devient le conte de Perrault dans cette mécanique parfaitement huilée ? Un peu plus tout de même qu'un simple prétexte, parce que le sel de l'épisode vient précisément du fait qu'il n'est nullement besoin de faire violence à sa mémoire pour trouver ses repères dans une histoire archi-connue dont le charme, indépendamment du déroulement à venir de l'épisode, tient d'emblée au pur plaisir de la répétition. L'héroïne le dit elle-même dès la première case : « les bonnes choses, c'est terrible, on ne s'en lasse jamais ». De fait, ne voir que de l'ironie dans ce propos liminaire serait réducteur. Certes, il est inutile de chercher des sens cachés aux contes, comme nous y enjoint la coccinelle qui, après avoir vainement tenté définir ce que ce récit était « au fond", renonce à cette impossible recherche de profondeur. Mais pourquoi ne pas se contenter de cette compulsion répétitive qui est, comme le rappelait Freud, l'une des plus pures jouissances de l'enfance?

23 L'analyse de «Trou de mémoire » confirme donc ce que l'on soupçonnait dès le début: il se dégage de l'ensemble des reprises de contes gotlibiennes une tendresse évidente pour les plaisirs de l'enfance, solidaire de la nostalgie qui imprègne d'autres épisodes 
de la $R A B$, et où le rire franc peut le céder à une poésie attendrie. Ainsi l'épisode relativement tardif de "La boule» $(R A B, 4,68-69)$ illustre-t-il une angoisse de la paternité qui semble bien réelle chez notre auteur: se réveillant un matin avec un boulet attaché au pied, Gotlib montre sa propre fille, bébé, entreprenant bravement de scier la chaine du boulet; mais cette libération ne dure qu'un temps, car dans les deux dernières images, on voit, par un effet de zoom très cinématographique, la pupille de l'enfant, maintenant âgée de trois ans, s'identifier à l'indécrottable boulet.

Gotlib joue donc avec les stéréotypes et les intrigues des contes, mais, au-delà des déformations, l'aura attachée à ceux-ci demeure souvent intacte. Et si « Sade expliqué aux enfants » met le doigt sur la réelle cruauté du texte de Perrault, c'est précisément parce que Gotlib le prend - paradoxalement, dira-t-on - au pied de la lettre : de toutes les planches que nous avons citées de la $R A B$, ce sont d'ailleurs sans doute les seules qui articulent des reproches explicites au contenu manifeste d'un conte. C'est pourtant là l'une des toutes premières histoires de la $R A B$, signe sûr que, si, comme on l'a rappelé, la série connaît au cours de ses cinq tomes de notables évolutions, la vision du conte de fée échappe en grande partie à ce processus. Au point que, nonobstant l'évolution du graphisme de l'auteur, «Incident de parcours ", qui clôt, dans le tome 5, notre premier corpus, aurait pu figurer sans rupture de ton, parmi les récits du tome 1. De fait, les histoires de fées s'amusent beaucoup plus à varier un imaginaire infiniment ductile qu'à ridiculiser un thème dont la fascination et l'efficacité narrative ne sont guère remises en question.

Il n'en va cependant plus tout à fait de même dans les trois tomes de Rhââ Lovely (RL) et les deux de Rhâ Gnagna $(R G)$ dont les premières éditions, s'égrenant de 1976 à 1980 reprennent des récits de longueurs très variables parus à partir de 1972 dans L'Écho des Savanes, puis dans Fluide Glacial. Seuls deux récits s'inspirent à proprement parler de notre corpus de contes ${ }^{15}$ :

- "Au p'tit bois-p'tit bois charmant quand on y va on est à l'aise » (RL, 1, réédition intégrale, 11-21): on y voit le maire d'une petite commune française confronté à la nécessité de raser le «Bois-Huon » pour construire des habitations de haut standing; s'endormant sur le dossier, il se retrouve dans le bois en question et y fait d'étranges rencontres : le Petit Poucet et ses frères se masturbent pour passer le temps, le loup et le Petit Chaperon rouge font la bête à deux dos, M. Seguin sodomise sa chèvre, Jean Valjean se fait faire une gâterie par Cosette, Blanche-Neige partouze avec les sept nains, et Tarzan soulève son pagne par seule concentration mentale; un deuxième rêve confronte le maire à l'enfant qu'il était, lequel le traite de "salaud» et se fait sauvagement réduire au silence par son double adulte.

- «Misère de misère " $(R G, 1$, ibid., 211-213) : réécriture de "La Petite Marchande d'allumettes ", hybridée avec la scène de la rencontre de Cosette et de Jean Valjean dans Les Misérables : c'est ainsi avec le seau de Cosette que Jean Valjean éteint l'incendie provoqué par l'héroïne d'Andersen, ce dont les deux fillettes ne lui sont guère reconnaissantes, la première ayant perdu son seau et la seconde ses allumettes.

26 C'est assurément le premier de ces récits qui réserve aux contes de fées le sort le plus radical. Cependant, la focalisation sur le maire, présenté comme un adulte rigide ayant profondément refoulé l'esprit de son enfance, laisse au lecteur la possibilité de considérer que la sexualisation outrancière dont sont victimes les contes n'est en fait que le résultat de l'esprit perverti de l'adulte qui en rêve. 

allusions sexuelles, les procédés de la $R A B$ : hybridation, progression délirante, trivialisation. Ici encore, en fin de compte, c'est le potentiel narratif des hypertextes sollicités plus qu'une volonté délibérée de désacralisation qui gouverne la pratique gotlibienne: même sa version de L'Exorciste n'est pas beaucoup plus grandguignolesque que le film original, et «Barbaralice » propose une réflexion subtile sur l'impensé du récit de Lewis Carroll (le désir d'être adulte de l'héroïne).

En conclusion, on se gardera donc de classer Gotlib dans la catégorie trop stricte de la "parodie ${ }^{16}$. Jusque dans les récits tardifs et fortement sexualisés de $R L$ et de $R G$, la composante nostalgique reste fortement présente, comme en témoigne la mine défaite du maire vieilli dans la dernière image de "Au p'tit bois-p'tit bois charmant... ». Audelà de la désacralisation, Gotlib utilise donc d'abord les contes parce qu'ils sont un trésor culturel commun et que le dessinateur est sûr que les allusions qu'il y fera seront comprises de chacun.

Comme on l'a suggéré plus haut, la grande préoccupation de Gotlib, dont l'œuvre connaît son acmé au tournant des années 1960, est d'abord d'interroger les limites du récit. Le gag en $\mathrm{BD}$ qui, jusqu'à lui, construisait immanquablement une narration entièrement orientée vers sa «chute " (comme un sonnet s'achemine inexorablement vers sa "pointe ») devient proliférant: les occasions de s'esclaffer sont partout, les marges envahissent le centre (à la manière de la fameuse petite coccinelle qui tente toujours de monopoliser, avec un succès mitigé, l'attention du lecteur), la conclusion perd son rôle focal et organisateur. On ne saurait, au fond, imaginer démarche plus structuraliste : l'espace dessiné n'est plus organisé à travers une " histoire ", mais par le biais des seuls jeux de forces et de tensions qui gouvernent les associations signifiantes, dont la collision génère cet humour "glacé et sophistiqué", pour reprendre l'expression récurrente de Gotlib ${ }^{17}$, qui inclut ainsi l'auto-dérision de son propre procédé dans l'incessante dérive du sens. Mais pour obtenir ce résultat, Gotlib a précisément besoin de s'appuyer sur un fond de références culturelles dont l'essence « narrative » est garante de la possibilité de les déconstruire.

Contrairement à celui de son ami et complice Mandryka qui, avec Le Concombre masqué, invente un univers parallèle dont l'apparente absurdité renforce en fait l'autonomie et la valeur allégorique (rien de moins gratuit que cette dénonciation lacano-marxiste de la société de consommation), le monde de Gotlib n'a pas de fond: toute la culture populaire, au sens large du terme ${ }^{18}$, s'y reflète dans une sarabande sans fin qui n'a pour but que de nous rappeler que l'on ne peut jamais rien inventer ${ }^{19}$, et que même les souvenirs personnels (d'où l'obsession gotlibienne de l'auto-représentation) sont toujours déjà pris dans un jeu de références et de stéréotypes qui en condamnent par avance la prétention à l'authenticité. À cet égard, les contes de fées, même s'ils ne représentent qu'un élément du vaste imaginaire collectif sollicité par Gotlib, peuvent apparaitre comme l'un des ressorts essentiels de la démarche de notre auteur : par le rapport qu'ils induisent avec une enfance fatalement introuvable, ils nous mettent sur la piste de la seule authenticité possible postulée par Gotlib, laquelle n'est, de fait, jamais qu'un paradis perdu. 


\section{BIBLIOGRAPHIE}

ERLBAUM, Lara, De l'autre côté de l'adulte - la parodie chez Marcel Gotlib ou le mélange de la culture savante et de la culture populaire, Université libre de Bruxelles, 1994-1995.

GARNER, James Finn, Politiquement correct : contes d'autrefois pour lecteurs d'aujourd'hui, trad. de l'anglais (USA) par Daniel Depland, Paris, Grasset, 1995.

-, De plus en plus politiquement correct : nouveau contes d'autrefois pour lecteurs d'aujourd'hui, trad. de l'anglais (USA) par Daniel Depland, Paris, Grasset, 1996.

GAUMER, Patrick, Les Années Pilote 1959-1989, Paris, Dargaud, 1997.

GoscinNY, René, Tous les visiteurs à terre, Paris, IMAV Éditions, 2010.

GoscinNY, René, GOTLIB, Marcel, Les Dingodossiers, 3 vols, Paris, Dargaud, 1967, 1972, 1995.

GOTLIB, Marcel, Rubrique-à-Brac, 5 vols, Paris, Dargaud, 1970-1974.

—, Rhâââââ... Lovely et Gnagna. L’Intégrale, Paris, Éditions Audie, 2004.

-, J'existe, je me suis rencontré, Paris, Flammarion, 1993.

-, Ma vie-en-vrac (entretiens avec Gilles Verlant), Paris, Flammarion, 2006.

MANDRYKA, Nikita, Le Concombre masqué. L'intégrale des années Pilote, Paris, Dargaud, 2004.

SADOUL, Numa, Gotlib, Paris, Albin Michel, 1974 (coll. « Graffiti »).

SANGSUE, Daniel, Le récit excentrique, Paris, Corti, 1987.

-, La relation parodique, Paris, Corti, 2007.

VIDAL, Guy, GoscinNY, Anne et GAUMER, Patrick, René Goscinny profession : humoriste, Paris, Dargaud, 1997.

\section{NOTES}

1. Remarquons que dans son autobiographie J'existe, je me suis rencontré et dans ses entretiens avec Gilles Verlant, Ma vie-en-vrac, Gotlib est loin d'idéaliser son enfance.

2. Publiés dans le magazine Pilote entre 1968 et 1972, et repris en album entre 1970-1974, les épisodes de la $R A B$ ont souvent deux titres : l'un intégré à la planche originale et un deuxième en caractère typographiques en haut de la page de la réédition en album. Même s'il est visiblement second dans l'intention de l'auteur, c'est toujours le titre de la réédition que je citerai en premier, ayant renoncé, pour ne pas surcharger les références, à mentionner la date des premières publications dans le journal Pilote. Certes, l'ordre des épisodes dans les albums ne correspond pas toujours rigoureusement à la chronologie des publications originales, mais c'est là une question de détail, car la succession des cinq tomes de l'édition définitive de la $R A B$, qui n'est décalée de la publication périodique que de deux ans à peu près, reflète suffisamment bien l'évolution de l'œuvre gotlibienne pour que mon propos n'en soit pas biaisé.

3. Voir G. Vidal, A. Goscinny et P. Gaumer, René Goscinny profession : humoriste.

4. Qui s'exprime bien dans son unique roman, Tous les visiteurs à terre, où il dresse des portraits savoureux des habitués des croisières transatlantiques. 
5. Geste complaisamment glosé par N. Sadoul, Gotlib, qui construit sa description de l'œuvre gotlibienne sur une interprétation psychanalytique de son évolution : le dessinateur effectuerait progressivement le meurtre du père de substitution qu'est Goscinny.

6. Voir P. Gaumer, Les Années Pilote.

7. Voir J. F. Garner, Politiquement correct et id., De plus en plus politiquement correct.

8. Voir «La Fontaine revu et corrigé » $(R A B, 1,28-29)$, reprise presque littérale de la fable «Le Savetier et le financier », augmentée d'un épilogue, et «Encore ce bon La Fontaine » ou «La Cigale et la fourmi » $(R A B, 1,72-73)$ qui montre la cigale se reconvertissant dans la pantomime.

9. Voir « Le Matou matheux » $(R A B, 2,65-72)$, hybridation des univers d'Alice au Pays des merveilles et du Philémon de Fred, et « La marionnette infernale » (RAB, 2, 89-96), hybridation de Pinocchio et de Frankenstein.

10. Plus anecdotique encore, on pourrait citer le troisième des onze tests de "Mesurez votre Q.I. » $(R A B, 1,36-37)$ qui consiste à retrouver la fin du « Petit Chaperon rouge ». On aura compris que mon recensement n'est pas exempt de tout reproche : quelques discrètes allusions féeriques dans les fourmillantes pages gotlibiennes peuvent encore m'avoir échappé.

11. Si son ami Mandryka, auteur du Concombre masqué, est bien connu pour être un grand lecteur de Freud, Marx et surtout Lacan (voir sa caricature dans $R A B, 5,64$ ), on chercherait en vain la moindre trace directe de lectures des structuralistes par Gotlib; ce qui n'a pas empêché Eric Loret d'écrire dans son hommage nécrologique à l'auteur de la Rubrique-à-brac que «c'est avec Gotlib que toute une génération a appris l'intelligence dans les années 70. Presque aussi bien que Roland Barthes, en plus rigolo tout de même » (Libération, 4 décembre 2016, consultable sur : <http://next.liberation.fr/culture/2016/12/04/gotlib-gailuron-delure_1532909>).

12. Pour prendre des épisodes évoqués dans la note 9 , Alice au pays des merveilles et l'univers de Fred sont sans doute plus proches que Pinocchio et Frankenstein - quoique...

13. Allusion probable, ici encore, à l'« Oncle Paul » du journal de Spirou, toujours représenté avec son immuable pipe. Notons en passant que la représentation du Petit Chaperon rouge stressé et du loup philosophe reconduit une caractérisation sexiste : la femme perd ses nerfs, mais l'homme (enfin, le loup...) sait se maîtriser.

14. Voir D. Sangsue, Le récit excentrique, qui analyse des textes du XIXe siècle dont le déroulement capricant et biaisé tourne le dos à la logique du récit réaliste promu à la même époque par Balzac. 15. On listera tout de même rapidement ici les autres récits de $R L$ et $R G$ qui évoquent le monde de l'enfance : "L'Exorcisme » (RL, 3, 155-171), réécriture gore du film L'Exorciste ; «La folle nuit » $(R G, 1,185-191)$, où deux enfants en font voir de toutes les couleurs au Père Noël ; « Barbaralice » ( $R G, 1,214-229)$, hybridation d'Alice au pays des merveilles et de la Barbarella de Forest; " Le membre fantôme » $(R G, 2,255-261)$, qui raconte les mésaventures, au Paradis, de la tête de saint Denis, malencontreusement confondue avec leur ballon par les deux sales gosses que sont saint Ouen et saint Cloud, et « Le Petit Prince » $(R G, 2,276-287)$, réécriture du conte de Saint-Exupéry avec un protagoniste à la fois demeuré et mal embouché.

16. D. Sangsue (La relation parodique) ne nous contredirait certainement pas.

17. Voir par ex. $R A B, 1,45$.

18. De ce point de vue, le seul titre de la thèse inédite de L. Erlbaum, De l'autre côté de l'adulte - la parodie chez Marcel Gotlib ou le mélange de la culture savante et de la culture populaire inspire des réticences : la «culture savante » ne me semble jouer à peu près aucun rôle chez Gotlib, et si l'on veut absolument désigner par là les Contes de Perrault où les Fables de La Fontaine, leur traitement est précisément celui que Gotlib réserve à la culture «populaire ", du cinéma à la vulgarisation zoologique, en passant par le roman policier et la psychologie de comptoir.

19. Deux démonstrations inverses mais concordantes: la fable express du scénariste qui réinvente Grimm sans le savoir, mais à qui on le fait vertement remarquer ; le conte « $Y$ en a être une fois » qui n'est autre que « Le Petit Chaperon rouge », mais que Gotlib feint de nous présenter comme authentiquement africain. 


\section{RÉSUMÉS}

Dans sa Rubrique-à-Brac, mais aussi dans ses bandes dessinées plus « adultes » (Rhâ-Lovely et RhâGnagna), Marcel Gotlib fait un large usage des contes de fées, et plus largement de tout un imaginaire culturel lié à l'enfance et à l'adolescence. La notion de " parodie » est loin d'épuiser les modalités de ce réemploi qui tient plus profondément à une interrogation multiforme sur la notion même et la possibilité du récit en bande dessinée.

In his Rubrique-à-Brac, but also in his more "adult" comics (Rhâ-Lovely and Rhâ-Gnagna), Marcel Gotlib makes extensive use of fairy tales, and more generally draws on a whole cultural imaginary linked to childhood and adolescence. The notion of "parody" is too limited to account for the manifold aspects and modalities of this re-use, which is deeply rooted in a multifaceted questioning of the very notion and possibility of the comic strip narrative.

\section{AUTEUR}

\section{ALAIN CORBELLARI}

Universités de Lausanne et de Neuchâtel 